\title{
THE IMPACT OF ADDITIVE NOISE ON POLARIMETRIC RADARSAT-2 DATA COVERING OIL SLICKS
}

\author{
Martine M. Espeseth, Stine Skrunes, Camilla Brekke, and Malin Johansson
}

\author{
UiT The Arctic University of Norway, Department of Physics and Technology, Tromsø, Norway
}

\begin{abstract}
We attempt to understand how a set of well known polarimetric Synthetic Aperture Radar (SAR) features are impacted by the additive system noise for mineral oil and produced water slicks. For this, we use quad-polarimetric SAR scenes from Radarsat-2. Oil slicks at sea can be detected using SAR instruments, and the dual- (HH-VV) and quad-polarimetric modes can provide additional information about the characteristics of the oil. Therefore the increase in polarization dimensionality may be beneficial in a potential clean-up situation. For example, characterization could aid in separating different types of oil slicks, like mineral oil and produced water as studied here. Oil slick characterization using scattering properties can only be performed if the returned signal is well above the noise floor. To avoid misinterpretation it is important to understand how the noise impacts the measured radar signal. Most of the features investigated in this study were to a larger degree influenced by the additive noise. Further, a backscatter signal level of $10 \mathrm{~dB}$ above the noise floor is identified as necessary to support analysis of the scattering properties within the oil slicks without too much noise contamination of the signal. The mineral oils and produced water slicks showed similar polarimetric behavior, despite their chemical and physical differences at release.
\end{abstract}

Index Terms - Synthetic Aperture Radar, Radarsat-2, polarimetry, additive noise, SNR, mineral oil, produced water

\section{INTRODUCTION}

It is well known that spaceborne Synthetic Aperture Radar (SAR) can be used for oil spill detection and ocean surveillance, which is already a common practice within operational monitoring services. Oil is detected due to the lowbackscatter response from the oil slicks compared to the surrounding clean sea. These detections can be of both legal and illegal spills from ships, rigs, etc,. An example of legal releases is produced water, which contains a small amount of mineral oil [1], that is discharged from production platforms and contain various constituents depending on the age and the type of oil well [2]. Previous studies have investigated the

This research is financed by CIRFA (RCN Grant no. 237906) and OIBSAR/Petromaks-2 (RCN Grant no. 280616). potential of quad-polarimetric SAR system for either separate mineral oil slicks from biogenic slick (see e.g., [3]) or extracting physical or chemical information from the oil slicks (see e.g., $[4,5])$. Oil slick characterization can only be performed if the returned signal is well above the noise floor. If this is not met, misinterpretation and misleading conclusions might occur.

Scaling, spatial correlation, and bias in the measured radar-cross-section (RCS) are three imaging effects that should be considered when attempting to recover the target RCS [6]. The bias, also known as the additive noise power, is investigated in this study. To avoid misinterpretation of the scattering properties it is crucial to have knowledge of how the random additive noise alters the measured radar signal. There are some previous studies that have discussed system noise in SAR data covering oil slicks [5, 7, 8]. For example, the effect of system noise on detections of oil slick using a set of features was studied in [7]. In this study, the goal is to confirm these suspicions by demonstrating how the additive system noise impacts a set of well known polarimetric features derived from measurements of either mineral oil or produced water slicks.

\section{ADDITIVE NOISE IN SAR}

The measured scattering matrix, $S^{m}$, is a function of the target scattering matrix [9], $\boldsymbol{S}$, i.e.;

$$
\boldsymbol{S}^{m}=\boldsymbol{S}+\boldsymbol{N}
$$

where $\boldsymbol{S}=\left[S_{H H}, S_{V H}, S_{H V}, S_{V V}\right]^{T}$ are the target scattering coefficients without noise, and $\boldsymbol{N}=\left[n_{H H}, n_{V H}, n_{H V}\right.$, $\left.n_{V V}\right]^{T}$ represents the complex additive noise, where $H$ and $V$ are horizontal and vertical polarizaton, respectively. $\boldsymbol{N}$ is assumed to be complex Gaussian white noise with zero mean, and the noise in the $\mathrm{HH}, \mathrm{VH}, \mathrm{HV}$, and $\mathrm{VV}$ polarization channels are assumed to decorrelate with each other [10]. The signal-to-noise ratio (SNR) in this study is estimated from the data and it is the ratio of the RCS and the nominal additive noise power given in the product file, i.e.;

$$
S N R_{p q}=\frac{\sigma_{p q}^{0}}{\sigma_{p q}^{n}}=\frac{\sigma_{p q}^{0, m}-\sigma_{p q}^{n}}{\sigma_{p q}^{n}}
$$


where $\sigma_{p q}^{0}$ is the RCS of the target, $p$ is the polarization on transmit, $q$ is the polarization on receive, and $\sigma_{p q}^{N}$ is the additive noise power, i.e., $\left|n_{p q}\right|^{2}$. The $\sigma_{p q}^{0, m}=\left\langle\left|S_{p q}^{m}\right|^{2}\right\rangle$ is the measured RCS, where $\langle\cdot\rangle$ denotes spatial averaging. A $9 \times 9$ averaging filter is applied to all scenes to reduce speckle. Note that, when $\mathrm{SNR}=1$ (or $\mathrm{SNR}=0 \mathrm{~dB}$ ) the additive noise and the backscattered power is equal. The noise-equivalentsigma-zero (NESZ) is defined in terms of the radar backscatter coefficient of an area that will appear at the same strength in the image as the background noise, i.e., $\mathrm{SNR}=1$. The NESZ (nominal values) is provided in the Radarsat-2 (RS-2) product file and is used as the noise power when estimating the SNR.

\section{MULTIPOLARIZATION PARAMETERS}

Table 1 shows the features investigated in this study, grouped into ratio-based or non-ratio-based feature categories. Using the two-scale Bragg models [11] [12], the small-scale ocean surface roughness is canceled in the ratio-based group, and the features are a function of the properties describing the surface tilts, incidence angle (IA), and dielectric constant. The polarization difference $(P D)$ and the real part of the copolarization cross-product $\left(r_{\mathrm{CO}}\right)$ are in the non-ratio-based group. The features in the ratio-based group are the copolarization ratio $\left(\gamma_{H H / V V}\right)$, magnitude of the copolarization correlation coefficient $\left(\rho_{C O}\right)$, the entropy $(H)$ and the mean $\alpha$ angle based on the $3 \times 3$ coherency matrix, the copolarization entropy $\left(H_{C O}\right)$ calculated from the $2 \times 2$ coherency matrix of the $\mathrm{HH}$ and VV channels, and the standard deviation of the copolarization phase difference $\left(\sigma_{\phi_{C O}}\right)$. Each of these features have been well studied in the literature, and some studies have established a certain interpretation of the behavior of each of these features for mineral oil slicks. Unfortunately, these interpretations might be questioned due to the influence of the additive noise component impacting the data. For example, low $P D$, low $r_{C O}$, high $\gamma_{H H / V V}$, low $\rho_{C O}$, high $H$, high $\alpha$, and high $\sigma_{\phi_{C O}}$ are typical values for oil-covered areas. In Section 5 we demonstrate that these "typical" values are strongly correlated with low SNR values, i.e., high additive noise power.

\section{DATA}

In this study, 15 RS-2 scenes acquired using the fine quadpolarimetric mode are used. Eight of these RS-2 scenes cover mineral oil slicks, and were acquired during oil-on-water exercises in the North Sea in the time period 2011-2016. The mineral oils were released under controlled conditions, and the oil slicks across the eight scenes have different properties and also weathers under different metocean conditions. The remaining seven RS-2 scenes cover produced water slicks. Produced water has much lower oil concentration than the mineral oil, and originates from controlled legal releases from
Table 1. Overview of the feature definitions. See e.g., [13] for the definitions of the pseudo probabilities $\left(p_{i}\right)$. The $\phi_{H H}$ and $\phi_{V V}$ are the phases of the complex scattering vectors $S_{H H}^{m}$ and $S_{V V}^{m}$.

\begin{tabular}{l|l} 
Non-ratio-based & Ratio-based \\
\hline$P D=\sigma_{V V}^{0, m}-\sigma_{H H}^{0, m}$ & $\gamma_{H H / V V}=\sigma_{H H}^{0, m} / \sigma_{V V}^{0, m}$ \\
$r_{C O}=\Re\left(\left\langle S_{H H}^{m} S_{V V}^{\star, m}\right\rangle\right)$ & $\rho_{C O}=\frac{\left|\left\langle S_{H H}^{m} S_{V V}^{\star, m}\right\rangle\right|}{\sqrt{\left\langle\left|S_{H H}^{m}\right|^{2}\right\rangle\left\langle\left|S_{V V}^{m}\right|^{2}\right\rangle}}$ \\
& $H=-\sum_{i=1}^{3} p_{i} \log _{3} p_{i}$ \\
& $H_{C O}=-\sum_{i=1}^{2} p_{i} \log _{2} p_{i}$ \\
& $\alpha=\sum_{i=1}^{3} p_{i} \cos ^{-1}\left(\left|\boldsymbol{e}_{\boldsymbol{i}}(1)\right|\right)$ \\
& $\sigma_{\phi_{C O}}=s t d\left(\phi_{H H}-\phi_{V V}\right)$
\end{tabular}

an oil platform, which is also located in the North Sea. Fig. 1 shows one example of the VV-intensity image of one mineral oil slick and one of the produced water slicks. Regions of interests (ROIs) are also shown in Fig. 1, where the blue ROIs represent the clean sea and the yellow ROIs cover the slicks (mineral oil or produced water). 600 pixels are randomly selected from the ROIs when calculating the various polarimetric features. Note that for each slick ROI there is a clean sea counterpart with the same incidence angle span. The produced water has a completely different chemical composition compared to the mineral oils at release. However, as shown in Fig. 1 both slick types dampens the small-scale ocean ripples resulting in a lower backscatter return compared to the surrounding clean sea. Table 2 shows the details of the SAR scenes in terms of acquisition time and incidence angle. Note that several slick and clean sea ROIs are extracted for each scene. The mineral oil scene range from $29^{\circ}-49^{\circ}$, which is almost the same as for the scenes containing the produced water slicks, i.e., $28^{\circ}-41^{\circ}$.

\section{ANALYSIS AND RESULTS}

This section presents two ways of evaluating the impact of the system noise.

\subsection{Feature sensitivity to additive noise}

Fig. 2 shows the mean feature value plotted against the $\mathrm{SNR}_{H H}$ in $\mathrm{dB}$ for the produced water, mineral oil slick, and clean sea areas. The reason for selecting the HH channel is because it generally produces lower RCS values than the $\mathrm{VV}$, making it more sensitive to additive noise. A logscale is used on the y-axis in the panels showing the $r_{C O}$ and $P D$ features for better visibility. All the features have to some degree a correlation with the additive system noise. The $\gamma_{H H / V V}$ seems to be the least influenced by the system noise. As expected, high entropy ( $H$ and $H_{C O}$ ) and high $\alpha$ values are observed for the slick-covered markers (produced 


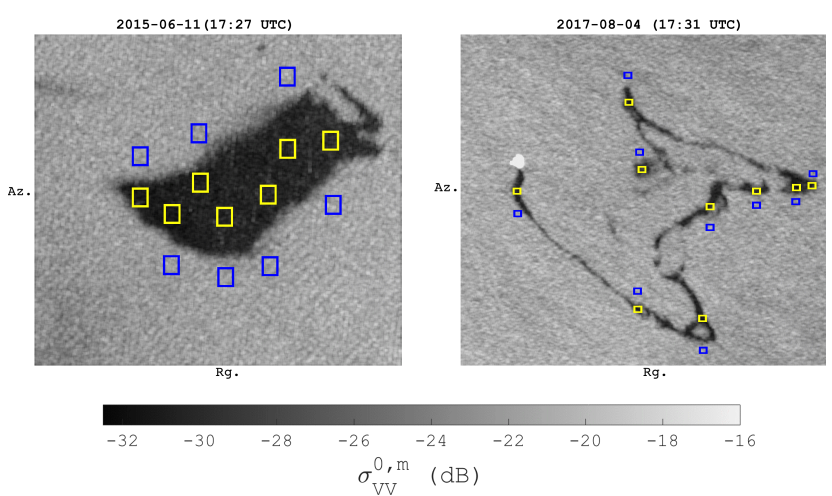

Fig. 1. Left panel: VV-intensity of one RS-2 scene covering mineral oil from the oil-on-water exercise in 2015. Right panel: VV-intensity of one RS-2 scene covering produced water from an oil platform (the white region in the right panel). RS-2 Data and Products MDA LTD. (2015,2017)(c)All Rights Reserved. Note that the size of the ROIs are different between the right and left panel.

water and mineral oil) at extremely low SNR values. This might be a result of the high noise power dominating the data. Further, the $\sigma_{\phi_{C O}}$ is also larger for the slick markers (orange and red) compared to the clean sea markers. The phase of the random noise has a uniform distribution resulting in a high $\sigma_{\phi_{C O}}$ value. Therefore, the high $\sigma_{\phi_{C O}}$ is most likely due to the low signal level and not the different scattering properties between the slicks and the clean sea. Based on Fig. 2, we observe that all the features, except the $\gamma_{H H / V V}$ are extremely sensitive to additive noise. Hence, care must be taken if these are used to interpret the scattering properties within both types of slicks (mineral oil and produced water). Further, approximately the same feature values are observed between the mineral oil and produced water slicks. Even though the SNR is low due to the low backscatter response within the oil slick, detection might still be possible as long as a high oil-sea contrast is given.

\subsection{Additive noise in $\mathbf{H} / \alpha$}

Fig. 3 contains $H / \alpha$ plots of the produced water and mineral oil areas. The Bragg scattering region [14] is indicated with a box, and the histogram of the markers inside (blue) and outside (red) this box is shown in the small panels. Bragg scattering is also observed within the oil slick according to [5, 7]. A departure from the Bragg scattering region towards the volume scattering region, when $\alpha$ is approximately in the interval $45^{\circ}-60^{\circ}[14]$, may be due to the high additive noise level in the data, i.e., low SNR. This is exactly what is observed in Fig. 3, where the markers with low SNR (orange - red) are located close to or within the volume scattering region. Hence, the departure from the Bragg region for the mineral oil and the produced water slicks happens when $\mathrm{SNR}_{H H}$ is approximately $10 \mathrm{~dB}$ (see the histograms in Fig. 3).
Table 2. Overview of the time of acquisition and mean IA of the SAR data used. The quad-polarimetric mode was used for all the C-band RS-2 scenes. RS-2 Data and Products MDA LTD. (2011-2013,2015-2018)-C)All Rights Reserved.

\begin{tabular}{cc|cc}
\multicolumn{2}{c|}{ Mineral oil } & \multicolumn{2}{c}{ Produced water } \\
\hline Date (Time UTC) & IA & Date (Time UTC) & IA \\
& $(\mathrm{deg})$ & & $(\mathrm{deg})$ \\
\hline 2013-06-11 (17:20) & $29^{\circ}$ & $2017-09-25(17: 15)$ & $28^{\circ}$ \\
$2012-06-15(06: 20)$ & $31^{\circ}$ & $2018-08-27(17: 14)$ & $28^{\circ}$ \\
$2011-06-08(17: 28)$ & $36^{\circ}$ & $2018-07-02(06: 19)$ & $30^{\circ}$ \\
$2015-06-11(17: 27)$ & $36^{\circ}$ & $2017-08-14(06: 11)$ & $37^{\circ}$ \\
$2016-06-15(17: 36)$ & $41^{\circ}$ & $2017-09-07(06: 11)$ & $37^{\circ}$ \\
$2016-06-15(06: 07)$ & $42^{\circ}$ & $2018-05-29(06: 11)$ & $37^{\circ}$ \\
$2011-06-08(06: 00)$ & $47^{\circ}$ & $2017-08-04(17: 31)$ & $41^{\circ}$ \\
$2012-06-15(17: 49)$ & $49^{\circ}$ & & \\
\hline
\end{tabular}

\section{CONCLUSION}

Low $P D$, low $r_{C O}$, high $\gamma_{H H / V V}$, low $\rho_{C O}$, high entropies ( $H$ and $H_{C O}$ ), high $\alpha$, and high $\sigma_{\phi_{C O}}$ are typical values for oil-covered areas, but are also, in general, expected values for surfaces that have a low SNR due to their low backscattering, as this study demonstrates. The same polarimetric behavior is observed for both the mineral oil and the produced water slicks. The feature response and the SNR values seem to be correlated, and care must be taken when interpreting the physical meaning, like scattering mechanisms, of the various features. We identify that the signal level should be at least 10 $\mathrm{dB}$ above the noise floor, to enable analysis of the scattering properties within the oil slicks without too much contamination from the additive noise in the system. Various multiplicative noise sources also have a significant negative impact on the SNR. This is investigated in an on-going study, which demonstrates how to include multiplicative noise sources in the estimation of the SNR.

\section{ACKNOWLEDGEMENT}

The Radarsat-2 data are provided by Norwegian Space Centre/KSAT under the Norwegian-Canadian Radarsat agreement 2011-2013,2015-2018.

\section{REFERENCES}

[1] G. K. Furnes, "Discharges of produced water from production platforms in the North Ltd Sea. Technical Report R-064641," Norsk Hydro, Bergen, Norway, 1994.

[2] C. O. Igwe, A. A. Saadi, and S. E. Ngene, "Opti- 

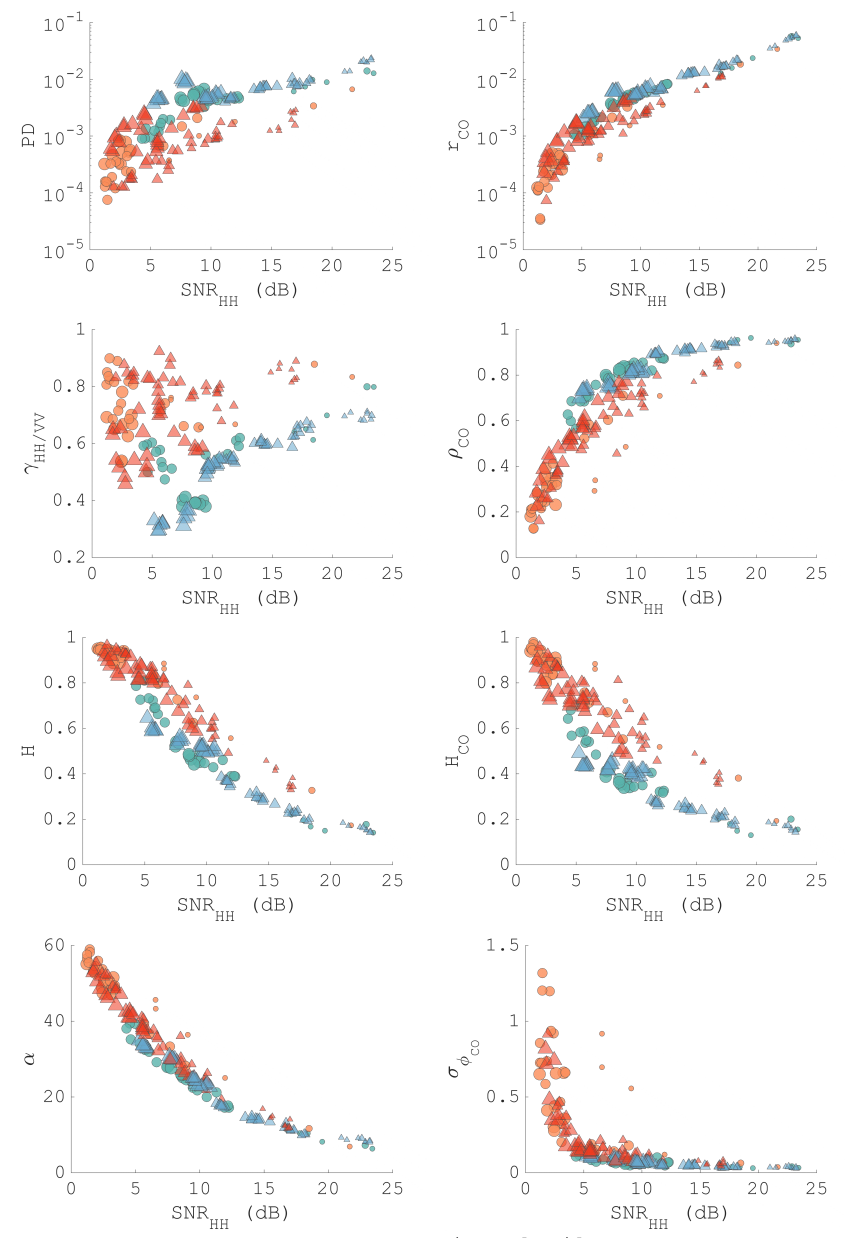

- Produced water - Clean Sea

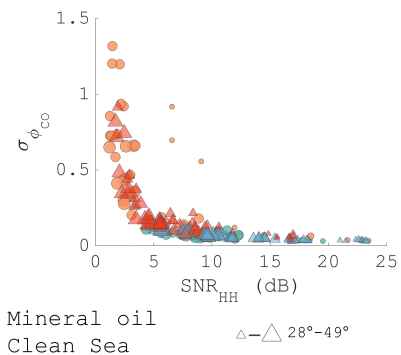

Fig. 2. Mean feature value as a function of the SNR in $\mathrm{HH}$ expressed in decibel $(\mathrm{dB})$. The marker size represents the IA value, ranging from $28^{\circ}-49^{\circ}$.

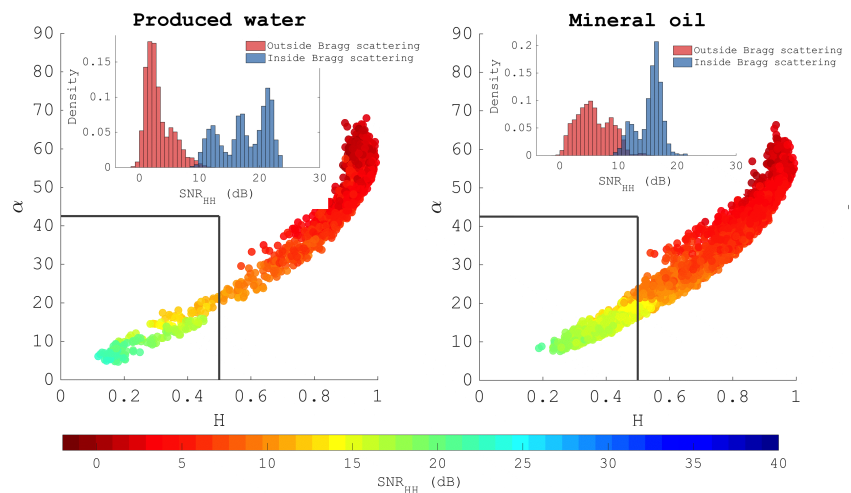

Fig. 3. $H / \alpha$ plots calculated from the produced water and mineral oil areas from the 15 scenes listed in Table 2. The small panels show the histograms of the areas inside (blue bars) and outside (red bars) the Bragg scattering region (the black box). mal options for treatment of produced water in offshore petroleum platforms," J. Pollut. Eff. Cont., vol. 1, no. 102, 2013.

[3] S. Skrunes, C. Brekke, and T. Eltoft, "Characterization of Marine Surface Slicks by Radarsat-2 Multipolarization Features," IEEE Trans. Geosci. Remote Sens., vol. 52, no. 9, pp. 5302-5319, Sept. 2014.

[4] S. Angelliaume, O. Boisot, and C.-A. Guérin, "DualPolarized L-band SAR Imagery for Temporal Monitoring of Marine Oil Slick Concentration," Remote Sens., vol. 10, no. 1012, pp. 1-17, Jun. 2018.

[5] B. Minchew, C. E. Jones, and B. Holt, "Polarimetric Analysis of Backscatter From the Deepwater Horizon Oil Spill Using L-Band Synthetic Aperture Radar," IEEE Trans. Geosci. Remote Sens., vol. 50, no. 10, pp. 3812-3830, Oct. 2012.

[6] C. Oliver and S. Quegan, Understanding Synthetic Aperture Radar Images. SciTech Publishing Inc., 2004.

[7] S. Angelliaume, P. C. Dubois-Fernandez, C. E. Jones, B. Holt, B. Minchew, E. Amri, and V. Miegebielle, "SAR imagery for detecting sea surface slicks: Performance assessment of polarization-dependent parameters," IEEE Trans. Geosci. Remote Sens., vol. 56, no. 8, pp. 1-21, Aug. 2018.

[8] W. Alpers, B. Holt, and K. Zeng, "Oil spill detection by imaging radars: Challenges and pitfalls," Remote Sens. Environ., vol. 201, pp. 133-147, Nov. 2017.

[9] S. R. Cloude, Polarisation Applications in Remote Sensing, First, Ed. Oxford University Press, 2010.

[10] A. Freeman, "The effects of noise on polarimetric SAR data," Geoscience and Remote Sensing Symposium, 1993. IGARSS '93. Better Understanding of Earth Environment., International, vol. 2, pp. 799-802, 1993.

[11] G. R. Valenzuela, "Theories for the interaction of electromagnetic and oceanic waves - a review," BoundaryLayer Meteorology, vol. 12, no. 1-4, pp. 61-85, 1978.

[12] I. Hajnsek, E. Pottier, and S. R. Cloude, "Inversion of surface parameters from polarimetric SAR," IEEE Trans. Geosci. Remote Sens., vol. 41, no. 4, pp. 727744, 2003.

[13] J.-S. Lee and E. Pottier, Polarimetric Radar Imaging, B. J. Thompson, Ed. CRC Press Taylor and Francis Group, 2009, no. 42-45.

[14] S. R. Cloude and E. Pottier, "An entropy based classification scheme for land applications of polarimetric SAR,” IEEE Trans. Geosci. Remote Sens., vol. 35, no. 1, pp. 68-78, Jan. 1997. 